noncritical sites. Although pathologic examination shows that specific plaque characteristics correlate with fatal ischemic events, plaques at high risk of rupture cannot be identified by conventional imaging techniques. Van Mieghem et al. assessed noninvasive identification of atherosclerotic plaques by multislice CT angiography compared with intravascular ultrasound (IVUS), the current gold standard technique. They then assessed the ability of two IVUS-based techniques - palpography and plaque ultrasonography - to identify high-risk atherosclerotic plaques in patients referred for percutaneous coronary intervention.

Compared with IVUS, multislice CT detected atherosclerotic plaque with $86 \%$ sensitivity and $69 \%$ specificity. On conventional IVUS, plaque dimensions at 6 months' follow-up stayed the same, and no alterations in the echogenicity of the plaques were detected. Palpography, however, enabled visualization of a wide prevalence of high-risk areas, and detected reductions in the number and density of high-strain spots between the baseline and 6-month measurements. The greatest reductions were observed in patients with acute myocardial infarction, the vast majority of whom had not received statins before presentation.

The authors urge caution in the use of noninvasive imaging for the detection of subclinical atherosclerosis because of the inability of the technique to detect dangerous lesions, which were found to be prevalent by palpography, even in cases of apparently mild disease. They also advocate intensive statin treatment aimed at reducing LDL cholesterol to levels below $1.8 \mathrm{mmol} / \mathrm{l}(70 \mathrm{mg} / \mathrm{dl})$ in patients with acute coronary syndromes.

Original article Van Mieghem CAG et al. (2006) Noninvasive detection of subclinical coronary atherosclerosis coupled with assessment of changes in plaque characteristics using novel invasive imaging modalities: the Integrated Biomarker and Imaging Study (IBIS). J Am Coll Cardiol 47: 1134-1142

\section{Folic acid and B vitamins fail to reduce major cardiovascular risk}

Observational data have indicated that raised homocysteine levels could increase cardiovascular risk. Plasma homocysteine is therefore a potential modifiable risk factor that could be readily controlled by daily supplementation with folic acid and vitamins $B_{6}$ and $B_{12}$.
The Heart Outcomes Prevention Evaluation (HOPE) 2 trial sought to determine whether long-term supplementation with folic acid and vitamins $B_{6}$ and $B_{12}$ could lower the major vascular event rate in individuals at high risk of cardiovascular events. In total, 2,758 participants were randomized to $2.5 \mathrm{mg}$ folic acid, $50 \mathrm{mg}$ vitamin $\mathrm{B}_{6}$ and $1 \mathrm{mg}$ vitamin $\mathrm{B}_{12}$ in a combined pill, and 2,764 were randomized to placebo, both administered daily.

After 2 years, individuals in the supplementation group had approximately double the folate and vitamin $\mathrm{B}_{12}$ levels, and approximately four times the vitamin $B_{6}$ levels, compared with levels at study start; the levels had not changed in the placebo group. Similarly, after an average of 5 years, the mean homocysteine level had decreased by $0.3 \mathrm{mg} / \mathrm{l}$ in patients receiving supplementation, whereas it had increased by $0.1 \mathrm{mg} / \mathrm{l}$ in patients receiving placebo. There was no difference in the composite endpoint of death, cardiovascular-related death or occurrence of myocardial infarction between the two groups; however, supplementation did reduce the stroke rate compared with placebo (111 [4.0\%] vs 147 [5.3\%] events; relative risk 0.75 , $95 \% \mathrm{Cl} 0.59-0.97 ; P=0.03$ ).

Although total homocysteine levels and stroke risk were reduced, mortality and myocardial infarction risk were not lowered and thus the authors do not recommend long-term folic acid and vitamins $\mathrm{B}_{6}$ and $\mathrm{B}_{12}$ supplementation.

Original article The Heart Outcomes Prevention Evaluation (HOPE) 2 Investigators (2006) Homocysteine lowering with folic acid and B vitamins in vascular disease. $N$ Engl J Med 354: $1567-1577$

\section{Intensive rosuvastatin therapy leads to regression of atherosclerosis}

Trials of atherosclerosis treatments have demonstrated halting of disease progression, as measured by intravascular ultrasound, but convincing evidence of regression has not been obtained. High-intensity statin therapy has had a more beneficial effect on cardiovascular outcomes than has been seen with more-moderate treatment. The prospective, open-label, blinded ASTEROID trial was, therefore, initiated to test the hypothesis that intensive rosuvastatin therapy, designed to achieve very low levels of LDL cholesterol, would lead to regression of 